\title{
1 WhichTF is dominant in your open chromatin data?
}

2 Yosuke Tanigawa ${ }^{1 *}$, Ethan S. Dyer ${ }^{2,3,7 *}$, Gill Bejerano ${ }^{1,4,5,6,+}$

3 1. Department of Biomedical Data Science, School of Medicine, Stanford University, Stanford, CA, USA.

4 2. Stanford Institute for Theoretical Physics, Stanford University, Stanford, CA, USA

5 3. Department of Physics and Astronomy, Johns Hopkins University, Baltimore, MD, USA

6 4. Department of Developmental Biology, Stanford University, Stanford, CA, USA.

7 5. Department of Computer Science, Stanford University, Stanford, CA, USA.

8 6. Department of Pediatrics, Stanford University School of Medicine, Stanford University, Stanford, CA, USA.

9 7. Current address: Google, Mountain View, CA, USA

$10 *$ These authors contributed equally and ordered by age

$11+$ Corresponding author (G.B. bejerano@stanford.edu)

12 Abstract

13 We present WhichTF, a novel computational method to identify dominant

14 transcription factors (TFs) from chromatin accessibility measurements. To rank TFs,

15 WhichTF integrates high-confidence genome-wide computational prediction of TF binding

16 sites based on evolutionary sequence conservation, putative gene-regulatory models, and

17 ontology-based gene annotations. Applying WhichTF, we find that the identified dominant

18 TFs have been implicated as functionally important in well-studied cell types, such as NF-

$19 \kappa \mathrm{B}$ family members in lymphocytes and GATA factors in cardiac tissue. To distinguish the

20 transcriptional regulatory landscape in closely related samples, we devise a differential

21 analysis framework and demonstrate its utility in lymphocyte, mesoderm developmental,

22 and disease cells. We also find TFs known for stress response in multiple samples,

23 suggesting routine experimental caveats that warrant careful consideration. WhichTF yields

24 biological insight into known and novel molecular mechanisms of TF-mediated

25 transcriptional regulation in diverse contexts, including human and mouse cell types, cell

26 fate trajectories, and disease-associated tissues. 


\section{Introduction}

28 Transcription factors (TFs) are the master regulators of development. They define,

29 refine, and can even divert cellular trajectories. TFs perform these important tasks by

30 binding to specific DNA sequences in open chromatin, where they recruit additional co-

31 factors and together modulate expression of downstream genes. TFs regulate biological

32 processes in healthy adult tissues, and mutations to both $\mathrm{TF}$ genes and their genomic binding 33 sites have been linked with human disease ${ }^{1,2}$.

The advent of next generation sequencing has paved the way for chromatin

35 immunoprecipitation followed by sequencing (ChIP-seq)-based methods for the discovery

36 of genome-wide loci where a given TF binds DNA in a given cell population ${ }^{3}$. Tools

37 developed for the analysis of ChIP-seq data, such as GREAT ${ }^{4}$ (Gene Regulatory Enrichment

38 of Annotations Tool), have discovered and leveraged a compelling phenomenon: when a TF

39 is functionally important for the progression of a certain process, such that its perturbation

40 leads to the disruption of this process, the binding sites for this TF are often highly enriched

41 in the gene regulatory domains of the "downstream" target genes that drive this process 4 .

TFs work in different combinations to enact a vast repertoire of cellular fates and

43 responses ${ }^{5}$. Between 1,500-2,000 TFs are thought to be encoded in the human genome ${ }^{1}$.

44 Performing ChIP-seq for more than a handful of TFs in any cellular context is an expensive

45 laborious procedure, while the assaying of hundreds of TFs even in the same cell state is

46 impractical except in a handful of settings, by the most lavishly funded consortia.

To obtain a more comprehensive view of transcriptional regulation in action,

48 experimental focus has turned from the assaying of individual TFs to the assaying of all

49 open chromatin in a given cellular context. These DNase-seq, ATAC-seq, or single-cell 
50 ATAC-seq accessibility profiles offer a proxy for all cis-regulatory elements active in a

51 given cellular state ${ }^{6-8}$.

52 While assaying all TFs is infeasible, many hundreds of TFs have been studied in one

53 or more cellular contexts, or via complementary methods (such as protein binding

54 microarrays or high-throughput SELEX), to obtain the DNA binding preference of the TF${ }^{1}$.

55 These hundreds of TF binding motifs can then be used to predict transcription factor binding

56 sites (TFBSs) for all characterized TFs in various context-specific sets of accessible

57 chromatin.

58 Very often, biological processes of interest are conserved at the genome sequence

59 level across closely related species, such as primates or mammals. As such, computational

60 tools like PRISM ${ }^{9}$ (Predicting Regulatory Information for Single Motifs) can be used to

61 obtain a rarefied subset of binding site predictions that are both observed to be positioned in

62 open chromatin and conserved orthologously in additional species. Because these sites

63 evolve under purifying selection, they are more likely to be individually important in the

64 probed context ${ }^{9}$.

Here, we innovate on the foundation of two tools our group previously developed:

$66 \mathrm{PRISM}^{9}$ for the prediction of evolutionarily conserved binding sites for hundreds of human and mouse TFs, and GREAT ${ }^{4}$ for the detection of functions enriched in gene regulatory

68 regions. We use insights from both to develop WhichTF, a tool that applies a novel

69 statistical test to identify the most dominant TFs within a set of user-specified open

70 chromatin regions. In this work, dominant TFs refer to TFs whose conserved binding sites

71 are enriched within functionally-coherent regions of the input open chromatin regions. We

72 show that our molecular definition of dominance successfully predicts biologically 
73 important factors in the context of different cell types, differentiation pathways, and even

74 disease associated cellular sets.

75 Results

\section{WhichTF Approach Overview}

77 In order to predict dominant TFs, WhichTF relies on both functional genome

78 annotations from GREAT and pre-curated, conservation-based predictions of TFBSs from

79 PRISM. As such, we use GREAT in conjunction with the mouse genome informatics (MGI)

80 phenotype ontology to annotate all genes in the human GRCh38 (hg38) and mouse

81 GRCm38 (mm10) genomes with a canonical transcription start site (TSS), a putative gene

82 regulatory domain, and any MGI phenotypes known to be affected by mutations to the

83 associated gene. This procedure yields more than 700,000 gene-phenotype relationships for

84 each genome (Fig. 1a, step 1) $)^{4,10-12}$. We also use PRISM to predict mammalian conserved

85 TFBSs using 672 manually curated PWMs from 569 TFs across the entire genome ${ }^{9}$. The

86 updated PRISM predictions resulted in 268 million and 161 million putative TFBSs for the

87 human and mouse genomes, respectively (Fig. 1a, step 2).

88 To confirm the utility of restricting ourselves to regulatory domains of highly

89 enriched ontology terms, we evaluated the relative enrichment in the number of TFBSs

90 within the input open chromatin region as a baseline method (Online Methods). We found

91 the baseline results are often overloaded with TFs associated with general housekeeping

92 processes (Supplementary Table S1). We therefore turned to focus on the top 100 enriched

93 terms (Online Methods). 
For a given query (Fig. 1a, step 3), WhichTF uses functional annotations to enhance

its prediction of dominant transcription factors. This is accomplished by computing TF

96 enrichments in only a restricted, particularly relevant, subset of the user's input. Specifically,

97 WhichTF uses GREAT to identify enriched ontology terms within the user's input query.

98 Each term is associated with a region of the genome corresponding to all of the regulatory

99 domains of genes annotated with that term. WhichTF selects the top 100 ontology terms.

100 For each term and every TF, WhichTF counts the number of binding sites falling in the

101 intersection of the user-specified accessible regions and the region of the genome associated

102 to the term of interest (Fig. 1a, step 4), and computes enrichment statistics, represented as a

103 TF-by-term enrichment matrix (Fig. 1b). Aggregating over the functional terms, WhichTF

104 computes a novel score and significance used for ranking TFs (Fig. 1c, Online Methods).

105 The top-ranked TFs are hypothesized to be functionally relevant TFs in a cell exhibiting the

106 indicated accessibility profile.

\section{WhichTF identifies functionally important TFs across diverse cell types}

108 To test the ability of WhichTF to identify functionally important TFs across different

109 cell types, we applied WhichTF to DNase-seq profiles and found that the predicted

110 dominant TFs are often confirmed to be functionally relevant by perturbation studies (Fig.

111 2a). In B- and T-cells, for example, we identified TFs in the NF- $\kappa$ B pathway, which are key

112 factors in lymphocyte development and adaptive immunity ${ }^{13}$. In embryonic heart tissue, we

113 found GATA-4, -5, and, -6 - known regulators of cardiac development and growth that,

114 when perturbed, have been implicated in human congenital heart disease ${ }^{14}$. In embryonic

115 hindbrain tissue, we found SOX2, a critical regulator of neural progenitor pluripotency and

116 differentiation in embryogenesis and later development, including adult hippocampal 
117 neurogenesis ${ }^{15-17}$. WhichTF yielded similar biologically meaningful results from the

118 corresponding cell types for mouse DNase-seq datasets (Supplementary Table S2),

119 suggesting that WhichTF can highlight both the identity and evolutionarily conserved

120 binding sites of key TFs from open chromatin in diverse contexts across species.

121 WhichTF robustly quantifies biologically meaningful similarities and differences in

122

123

124

125

126

128

130

131

\section{WhichTF identifies differentially dominant TFs for closely related cell types}

$$
\text { B-cells and T-cells share a closely related developmental trajectory }{ }^{13} \text {. As Fig. 2a }
$$

shows, WhichTF identified NF- $\kappa$ B family members NFKB1, RELA, and RELB as shared

dominant TFs. WhichTF also identified lineage-specific factors, such as SPI-B for B-cells

and RUNX3 for T-cells (Fig. 2a). SPI-B is an ETS family TF known to play a key role in B-

cell development and function, and environmental response ${ }^{19-21}$. RUNX3, in contrast, play

138 T-cell-specific functional roles, such as in CD4 versus CD8 thymocyte commitment, helper 
139 versus killer T-cell specification, and helper type selection ${ }^{22}$. These differential roles for

140 SPI-B and RUNX3 are corroborated by their cell-type-specific expression in B-cells and T-

141 cells, respectively $(\text { Fig. } 3 a)^{23}$.

142 Although we identified multiple TFs distinguishing B- and T-cells, the results are

143 dominated by common factors. This is reasonable, as they share most of their developmental

$144 \operatorname{program}^{13}$. To identify TFs with relative dominance from a given pair of samples, we

145 developed a differential analysis framework focusing on uniquely accessible regions only in

146 one sample (Online Methods). In B-cells, the differential analysis highlighted additional

147 ETS family members, PU.1 and SPI-C. These TFs are essential for healthy B-cell

148 differentiation and function (Fig. 3b). In T-cells, we saw an additional RUNX family

149 member, RUNX1, as well as $\operatorname{CBF} \beta$ (Fig. 3b) - both are functionally relevant in T-cells.

150 Indeed, RUNX1, RUNX3 and CBF $\beta$ form a complex and are crucial for the healthy function

151 of T-lymphocytes ${ }^{32}$.

\section{WhichTF identifies differentially dominant TFs along developmental trajectories}

153 TFs regulate cell fate decisions in animal developmental programs ${ }^{1}$. To gain insights

154 into the molecular mechanisms influencing cellular differentiation, we applied WhichTF to

155 ATAC-seq data from timepoints along mesoderm development to identify differentially

156 dominant TFs that distinguish cell fates at each step along the trajectory, from human

157 embryonic stem cells (ESCs) to early somite vs. cardiac mesoderm (Fig. 4) ${ }^{24}$.

158 The first step of mesoderm development is the differentiation from ESCs to anterior

159 (APS) or mid (MPS) primitive streak (PS) cells. In both APS and MPS cells, we found

160 WNT signaling TFs, such as TCF7L2 and LEF1, as well as T-box family TFs, such as TBX-

1612 and -3 (Fig. 4a-b). WNT signaling is involved in PS differentiation and is crucial in 
162 inducing PS cell types ${ }^{24}$. T-box family members also play key roles in PS development.

163 TBX6 is a canonical PS marker, and the specific loss of Eomes (a.k.a. Tbr2), causes ectopic

164 primitive streak formation in mice ${ }^{24,25}$. The specific T-box family member TBX3, ranked

165 third in APS cells, has been implicated in early stage of differentiation towards mesoderm

166 from ESCs in mouse and Xenopus and has been reported for its functional redundancy with

167 Tbx2 during Xenopus gastulation ${ }^{26}$. RUNX3, our top hit for APS, shows conserved

168 expression in mouse neuromesodermal progenitor (NMP) cells and human D3-NMP-like

169 cells. Interestingly, we also found previously unreported T-box family TFs, TBX15 and

170 TBR1, of which TBX15 is linked to decreased skeletal muscle mass in mouse ${ }^{12}$ and known

171 for tissue-specific expression in muscle, a tissue developed from the mesoderm lineage

172 (Supplementary Figure S1).

173 In paraxial mesoderm, we found WNT signaling TFs, which promote paraxial and

174 suppress lateral mesoderm (Fig. 4c) ${ }^{24}$. We also find HOXC13, necessary for proper

175 development of the paraxial mesoderm into the presomatic mesoderm ${ }^{27}$. In early somites,

176 we found MEIS2 and ZIC2, which are required in development of cranial and cardiac neural

177 crest and somite cells, respectively (Fig. 4 d) $)^{28,29}$.

178 In lateral mesoderm, we found multiple GATA family members, of which GATA4 is

179 a downstream effector of BMP signaling in lateral mesoderm (Fig. 4e $)^{30}$. We also saw

$180 R U N X 3$, which is co-expressed with $R U N X 1$ in lateral mesoderm ${ }^{31}$; both are necessary for

181 hematopoiesis 22,32 . GLI1, a key TF in hedgehog (HH) signaling, is necessary for

182 establishing left-right asymmetry in lateral mesoderm ${ }^{33}$. In cardiac mesoderm, we found

183 FOS TFs, GATA TFs, and GLI1 (Fig. 4f). Interestingly, FOSL2 regulates the rate of

184 myocardial differentiation ${ }^{34}$, and HH signaling via GLI1 is required for secondary heart 
185 field development ${ }^{35}$. As mentioned above, GATA factors are canonical drivers of cardiac

186 development and all the GATA family members identified for mesoderm development

187 (GATA-1, -2, -4, and -6) are implicated in Human cardiovascular diseases ${ }^{14,2}$.

188 WhichTF identifies potentially disease-relevant TFs

189 Transcriptional mis-regulation has a broad impact on human diseases ${ }^{2}$. To assess

190 whether WhichTF can shed light on the transcriptional regulatory molecular basis of human

191 disorders, we examined systemic lupus erythematosus (SLE) as a case study. SLE is a

192 heterogeneous and chronic autoimmune disorder most prevalent in young women and

193 affecting $0.1 \%$ of the population. Its genetic and epi-genetic bases are poorly understood

194 with known genetic associations accounting for only $10-20 \%$ of the observed heritability.

195 While SLE is characterized by mis-regulated immune response in T- and B-cells, few TFs

196 have been identified to play functionally relevant roles in $\mathrm{SLE}^{36}$.

197 To better understand the regulatory landscape of SLE, we identified differentially

198 dominant TFs in healthy B-cells compared to SLE-affected B-cells and vice versa by

199 applying WhichTF to ATAC-seq datasets ${ }^{37}$. We found BCL6 as a differentially dominant

200 TF in healthy vs. SLE B-cells (Table 1). BCL6 is an important marker of T-helper follicular

201 cells, a T-cell subtype which has been found to be mis-regulated in SLE ${ }^{38}$. Other

202 differentially dominant TFs and their corresponding genes are implicated in autoimmune

203 disorders (Table 1). A sonic hedgehog (SHH)-Gli signaling pathway member GLI1 is

204 involved in pathogenesis of rheumatoid arthritis through synovial fibroblast proliferation ${ }^{39}$.

205 A common genetic variant in $T C F 7 L 2$, which is known for type 2 diabetes risk allele,

206 discriminates autoimmune from non-autoimmune type 1 diabetes in young patients ${ }^{40}$. In a 
207 model system to study multiple sclerosis, ZEB1 is suggested as a regulator of experimental

208 autoimmune encephalomyelitis ${ }^{41}$.

WhichTF uncovers stress response signatures

210 Context-specific measurements of open chromatin typically require purification of

211 the desired cell type through mechanical and enzymatic tissue dissociation, which can be

212 quite taxing on the cells. Indeed, it has been reported that stress response factors are often

213 highly expressed in dissociated tissues ${ }^{42}$. Corroborating these observations, WhichTF often

214 identifies canonical stress-associated TFs as some of the most dominant TFs in multiple

215 very different contexts. As an illustration, we present WhichTF results for additional

216 DNase-seq datasets (Table 2). For three endothelial cell types and adrenal gland cells, we

217 found many members of FOS/AP-1 and NF- $\mathrm{kB}$ TFs, which are both known for their roles in

218 stress response. We also found ZFP410 (also known as ZNF410), a poorly characterized

219 Zinc finger TF, among the top hits across multiple cell types, suggesting its potential role in

220 stress response. Even in the samples dominated by stress-associated TFs, we still found

221 well-known context-specific players among the top hits, such as GATA3 and WT-1 in

222 kidney cells and SOX and FOX TFs in endothelial cells ${ }^{43-45}$. We also found that the

223 boundary between stress response and cell-type specific functions can be ambiguous, or at

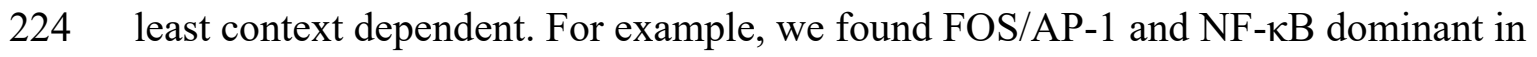

225 keratinocytes and B-cells, respectively which, in addition to being stress-associated, are also

226 known for their context-specific functions ${ }^{13,46}$. 
We present WhichTF, a novel computational method to identify and rank known or novel dominant TFs in any given set of accessible chromatin regions or through pairwise

230 differential analysis of related samples. The WhichTF score is built on high confidence

231 PRISM $^{9}$ predictions of conserved TFBSs as well as gene regulatory domain and ontological

232 annotation models from GREAT ${ }^{4}$. Applying WhichTF to dozens of samples across diverse

233 biological contexts, such as multiple cell types, developmental programs, and disease

234 samples, we found that the functional relevance of the identified dominant TFs is often

235 supported or suggested by published literature.

236 WhichTF identifies not only cell-type specific TFs, but factors reflecting biological

237 processes shared among multiple samples. One such example in our result, corroborated by

238 previous expression profiling, suggests stress response due to cellular dissociation is a

239 shared process ${ }^{42}$. In addition to previously identified factors, we report an under-

240 characterized Zinc finger protein, ZNF410, as a TF potentially involved in cellular stress

241 response. The identification of stress associated TFs suggests WhichTF may serve as a

242 useful quality control of chromatin accessibility data.

As we have demonstrated above, WhichTF is broadly applicable. WhichTF takes as

244 input any form of chromatin accessibility measurement for either human or mouse, the two

245 most studied genomes. Our illustrative examples span both species and assay types, such as

246 DNase-seq and ATAC-seq. When combined with emerging single-cell accessibility

247 profiling technologies ${ }^{8}$, WhichTF will provide systematic characterization of dominant TFs

248 across a spectrum of cell-types. For example, application of WhichTF to datasets from

249 large-scale projects, such as the Human Cell Atlas project ${ }^{47}$, has the potential to discover 
250 dominant TFs for each cell type and binding sites of those TFs. Moreover, our differential

251 analysis framework will help in understanding how closely related cell types diverge by

252 providing hypotheses of differentially important TFs.

253 The resources made available with this study, including WhichTF and the GREAT

254 update, provide an excellent foundation for investigating the molecular mechanisms of TF-

255 mediated cis-regulation. Together, these results highlight the benefit of combining

256 experimental characterization of chromatin accessibility, high-quality TFBS reference

257 datasets, and ontological genome annotation, suggesting that systematic identification of

258 dominant TFs across a large number of samples will be a powerful approach to understand

259 molecular mechanisms of gene regulation and their influence on cell type differentiation,

260 development, and disease. 
262 Online Methods

263 GREAT v.4.0.4 update

264 We performed a major update of Genomic Regions Enrichment of Annotations Tool

265 (GREAT) $)^{4}$ and released it as version 4.0.4. GREAT currently supports the human (Homo

266 sapiens GRCh38 and GRCh37/hg19) and mouse (Mus musculus GRCm38/mm10 and

267 NCBIM37/mm9) genomes. We obtained Ensembl gene sets from the following Ensembl ${ }^{48}$

268 versions:

269 - Human GRCh38: Ensembl version 90

270

- Human GRCh37: Ensembl for GRCh37 version 90

271

- $\quad$ Mouse GRCm38: Ensembl version 90

272

- $\quad$ Mouse NCBIM37: Ensembl version 67

273 By focusing on the set of genes with at least one Gene Ontology (GO) annotation ${ }^{10,11}$ as

274 described before ${ }^{4}$, we defined putative gene regulatory domains for 18,777 (GRCh38),

275 18,549 (GRCh37/hg19), 21,395 (GRCm38/mm10), and 19,996 (NCBIM37/mm9) genes'

276 canonical transcription start sites.

277 We also updated the ontology reference data. GREAT currently supports the most

278 recent versions of the following ontologies at the time of analysis: Ensembl genes, Gene

279 Ontology $(\mathrm{GO})^{10,11}$, human phenotype ontology $\mathrm{y}^{49}$, and mouse genome informatics (MGI)

280 phenotype ontology ${ }^{12}$ (Supplementary Table S3). The new Ensembl genes ontology is a

281 "flat" ontology that makes every gene into a term, facilitating the testing of cis-regulatory

282 elements congregation in the regulatory domains of individual genes. For MGI phenotype

283 ontology, we mapped MGI gene identifiers to Ensembl human gene IDs using one-to-one

284 orthology mappings from Ensembl Biomart ${ }^{48}$ version 90. In total, we compiled 2,861,656, 
2,846,384, 2,734,172, and 2,675,691 gene-term relationships for GRCh38, GRCh37,

GRCm38, and NCBIM37 genome assemblies, respectively (Supplementary Table S3).

\section{Computational TFBS prediction with PRISM}

To take advantage of growing sequence data from both multiple species and

289 functional genomics datasets, we updated our computationally predicted PRISM conserved

290 transcription factor binding sites (TFBSs) for the human (Homo sapiens GRCh38 and

291 GRCh37) and mouse (Mus musculus GRCm38 and NCBIM37) genomes. Briefly, PRISM

292 predicts TFBSs based on evolutionary conservation of TF motif matches ${ }^{9}$. The GRCh37 and

293 NCBIM37 tracks are derived using liftOver ${ }^{50}$ from that of GRCh38 and GRCm38,

294 respectively.

We used the following multiple alignment from the UCSC genome browser ${ }^{50}$ :

298 We removed Killer whale (Orcinus orca, orcOrc1) from the human alignment because of

299 chromosome name mismatch. We further subset the alignments to Eutherian species ${ }^{9}$,

300 resulting in 57 and 40 species for human and mouse, respectively. Using our manually

301 curated TF monomer motif library ${ }^{51}$, we applied PRISM ${ }^{9}$ with the default parameters and

302 focused on the top 10,000 predicted TFBSs for each TF in our analyses. We used GNU

303 parallel in our analysis ${ }^{52}$.

\section{Baseline TF enrichment method without functional annotation}

We computed the binomial p-value of each TFBS set, using the total number of

306 TFBS predictions, the number intersecting the query and the fraction of the genome covered 
307 by the open chromatin region. We ranked the TFs by their binomial fold (Supplementary

308 Table S1).

309 WhichTF analysis protocol

310 WhichTF combines user specified accessibility measures, such as ATAC-seq or

311 DNase-seq peaks with precomputed reference datasets to produce a ranked list of context

312 specific, dominant TFs. The reference datasets consist of GREAT regulatory domain models,

313 MGI mouse phenotype ontology-based gene annotations, and PRISM TFBS predictions.

314 WhichTF first identifies the top 100 ontology terms $\left(\pi_{1}, \ldots, \pi_{100}\right)$ based on the

315 GREAT enrichment test on the input query set with the default "basal plus extension"

316 association rule and a filter that terms must be associated with no fewer than two genes and

317 no more than 500 genes associated to them. For each TF in the PRISM TFBS prediction

318 library of $N$ TFs, WhichTF takes an intersection of the TFBS prediction track and the user

319 submitted open regions using overlapSelect ${ }^{50}$.

320 Each TF in the PRISM library has a different number of TFBSs and regulatory

321 domains of different total sizes associated with each term. To capture the relative

322 importance of different TFs within different contexts, WhichTF computes a few measures of

323 statistical significance for each transcription factor and term and summarizes these measures

324 in TF by term summary statistic matrices. Specifically, we apply hypergeometric and

325 binomial tests defined below: 

and $K_{i j}$ as follows:

- $n_{i}=\#\left\{\right.$ TFBS $_{i} \cap$ QUERY $\}$

- $k_{i j}=\#\left\{\left(\mathrm{TFBS}_{i} \cap \mathrm{QUERY}\right) \cap \operatorname{RegDom}_{j}\right\}$

- $\quad N=\#\left\{\left(\mathrm{U}_{k} \mathrm{TFBS}_{k}\right) \cap \mathrm{QUERY}\right\}$

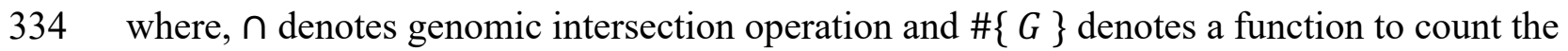

335 number of elements in genomic regions, $G$. With these parameters, we compute the

336 hypergeometric $\mathrm{p}$-value for each pair of $\mathrm{TF}_{i}$ and term $\pi_{j}$ :

$$
\sum_{k=k_{i j}}^{\min \left(n_{i}, K_{j}\right)} \frac{\left(\begin{array}{c}
K_{j} \\
k
\end{array}\right)\left(\begin{array}{c}
N-K_{j} \\
n_{i}-k
\end{array}\right)}{\left(\begin{array}{c}
N \\
n_{i}
\end{array}\right)}
$$
value for each pair of $\mathrm{TF}_{i}$ and term $\pi_{j}$ :

342 where, $p_{\pi}$ denotes the probability of drawing a base annotated with term $\pi$ from non-gap

343 genomic sequences under the uniform distribution ${ }^{4}$. 


\section{Adaptive TF significance threshold}

345

To eliminate false positives, WhichTF focuses on terms where the most significant

346 TF characterized by both hypergeometric and binomial p-value match. Using the enrichment

347 statistics, WhichTF selects dominant TFs for each selected ontology term. We compute the

348 adaptive threshold for each of the hypergeometric and binomial test by finding a leap in the

349 p-values of the top 10 TFs for each term using the following procedure. Let's denote the top

35010 hypergeometric $\mathrm{p}$-values for a fixed functional term $\pi$ as $p_{1} \leq p_{2} \leq \cdots \leq p_{10}$. We

351 define the difference of adjacent negative $\log$ of $\mathrm{p}$-values as $d_{k}=-\log \frac{p_{k}}{p_{k+1}}$. We define $m$,

352 the index with the largest leap in p-value as $m=\operatorname{argmax}_{k} d_{k}$. Our adaptive threshold is $p_{m}$

353 and we only keep TFs with hypergeometric $p$-values that satisfies $p \leq p_{m}$. We define the

354 adaptive threshold for binomial $\mathrm{p}$-values in the same way. We say $\mathrm{TF}_{i}$ is significant for term

$355 \pi_{j}$ when it passes the adaptive thresholds for both TF hypergeometric and TF binomial tests.

\section{WhichTF scores}

357 For each TF, WhichTF computes the score by the following equation. Let $\left(\pi_{1}, \ldots, \pi_{K}\right)$ be the

358 set of terms selected from step 1 in the order of relevance with $\pi_{1}$ as the top hit. Let

$359 \operatorname{Rank}\left(\mathrm{TF}_{\mathrm{i}}, \pi_{j}\right)$ be the rank of the $\mathrm{TF}_{\mathrm{i}}$ for term $\pi_{j}$. Let Significant $\left(\mathrm{TF}_{\mathrm{i}}, \pi_{j}\right)$ denote a Boolean

360 variable that indicates whether $\mathrm{TF}_{\mathrm{i}}$ passes the filters described above for term $\pi_{j}$ (i.e.

361 Significant is 1 if the TF passes the significance filter and zero otherwise). With this

362 notation, we define the WhichTF score of $\mathrm{TF}_{\mathrm{i}}$ as:

$$
\text { WhichTF score }\left(\mathrm{TF}_{i}\right)=\sum_{j} \frac{\operatorname{Significant}\left(\mathrm{TF}_{\mathrm{i}}, \pi_{j}\right)}{\sqrt{j \cdot \operatorname{Rank}\left(\mathrm{TF}_{\mathrm{i}}, \pi_{j}\right)}}
$$


365 WhichTF computes the statistical significance of a WhichTF score based on a null model

366 that any ordering of TFs within each term is equally likely. Thus, the probability of a given

367 score is determined by the relative number of configurations with the score. To enumerate

368 the number of configurations with a given score in polynomial time, we devised a dynamic

369 programing approach ${ }^{53}$ which acts recursively on the number of functional terms, $K$. This

370 procedure first discretizes each contribution to the summand in the definition of the

371 WhichTF score defined above. Let $\left\{s_{j 1}, s_{j 2}, \ldots, s_{j M_{j}}\right\}$ be the set of all the possible cumulative

372 scores up to term $\pi_{j}$, that is the scores gotten by computing the above sum only up to

373 term $\pi_{j}$. Here, $M_{j}$ is the number of distinct discretized scores up to term $\pi_{j .}$. Let $n_{j i}$ represent

374 the number of different ways of getting each such score, $s_{j i}$, and let $S_{j}=\left\{\left(s_{j 1}, n_{j 1}\right),\left(s_{j 2}\right.\right.$,

$\left.\left.375 n_{j 2}\right), \ldots,\left(s_{j M_{j}}, n_{j M_{j}}\right)\right\}$ be the set of all tuples of scores and number of configurations. Finally,

376 let $\left\{t_{j 1}, t_{j 2}, \ldots, t_{j M_{j}}\right\}$ denote the individual summands at term $\pi_{j}$.

377 The p-value of each score is computed directly from $S_{K}$, the full set of cumulative

378 scores and number of configurations, by dividing the number of configurations with scores

379 greater than or equal to a given score by the total number of configurations. This list of

380 tuples, $S_{j}$, can be computed recursively with the base case of $S_{0}=\{(0,1)\}$. The set of scores

381 at level $j+1$ is given by all combinations, $s_{j i}+t_{j+1 k}$, with the number of configurations

382 given by aggregating over all combinations of $s$ and $t$ that yield the same cumulative score.

383 Given that the WhichTF scores of multiple TFs are not independent, we apply the

384 procedure defined above from the top scoring TF to the TF with the lowest score and

385 compute conditional statistical significance. This means that for the computation of 
386 statistical significance of the $i$-th ranking TF, we remove TFs whose rank is smaller than $i$

387 and apply the recursive procedure defined above.

390 From the ENCODE/Roadmap data portal, we obtained "hotspot" files derived from DNase-

391 seq experiments ${ }^{54,55}$. All coordinates are provided in GRCh37. We present analysis spanning

39295 samples from 12 cell types and tissues (Supplementary Table S4).

393 We systematically applied WhichTF to each sample and obtained the ranked list of

394 TFs as well as a vector of WhichTF scores across all TFs in the library (Figure 2a, Table 2).

395 We applied t-SNE, a non-linear dimension reduction method ${ }^{18}$, implemented in Python

396 Scikit Learn library ${ }^{56}$ with perplexity 10 (Figure 2b).

Using mouse ENCODE DNase-seq datasets provided in GRCm38 from the four cell

398 types used for the human analysis (Figure 2a, Supplementary Table S5), we applied

399 WhichTF using mouse GRCm38 reference dataset (Supplementary Table S2).

Cell type-specific expression analysis

401 We presented cell type-specific RNA-seq data from the GEO database (GSE118165) ${ }^{23}$. We

402 subseted this dataset to the unstimulated samples and plotted the expression of SPIB and

$403 \quad R U N X 3$ for lymphoid cells in T and B cell lineages (Figure 3a). 
407 "subtract" to keep a subset of A that does not overlap with B. We applied WhichTF single

408 run mode (above) on the identified differentially accessible regions (Figure $3 \mathbf{b}$ ).

410 Using ATAC-seq datasets (SRP073808 from NCBI GEO database) of mesoderm

411 development ${ }^{24}$ (Supplementary Table S6), we applied WhichTF differential analysis

412 following the diagram of sequential differentiation (Figure 4).

\section{Systemic lupus erythematosus dataset}

414 Eight sets (4 SLE and 4 healthy controls [HC]) were taken from the NCBI sequence read

415 archive (SRA, Supplementary Table S7). Paired end reads were mapped using bowtie2

416 with the outer distance flag (-X) set to 1000 and otherwise default settings ${ }^{58}$. Samtools was

417 used to generate a sorted bam file and MACS2 was used to call peaks with shift set to 37 ,

418 extension size set to 72 and broad and keep-dup flags on ${ }^{59,60}$. Given that some of the

419 samples in this dataset are from a biobank, we conservatively defined differentially

420 accessible regions shown below and applied WhichTF differential analysis (Table 1):

- $\quad \mathrm{SLE}-\mathrm{HC}:=\mathrm{SRR} 3158183-\mathrm{U}_{x \in \mathrm{SRR} 3158176-9} x$

- $\mathrm{HC}-\mathrm{SLE}:=\bigcap_{x \in \mathrm{SRR} 3158176-9} x-\mathrm{U}_{x \in \mathrm{SRR} 3158180-3} x$

424 Using the data obtained from the GTEx Portal ${ }^{61}$ on 05/24/2019 (phs000424.v7.p2), we

425 investigated whether the identified TFs in have a tissue-specific expression

426 (Supplementary Figure S1). 


\section{Data availability}

428 All datasets analyzed in this study are publicly available through the ENCODE/Roadmap 429 portal [https://www.encodeproject.org/], NCBI GEO database

430 [https://www.ncbi.nlm.nih.gov/geo/], NCBI sequence read archive [NCBI sequence read

431 archive], or the GTEx Portal [https://gtexportal.org] with identifiers included in

432 Supplementary Tables S4-S7 and in Online Methods.

\section{Code availability}

434 WhichTF program and analysis scripts are available at our Bitbucket repository:

435 https://bitbucket.org/bejerano/whichtf

436 GREAT version 4.0.4: https://great.stanford.edu

\section{References}

438 1. Lambert, S. A. et al. The Human Transcription Factors. Cell 172, 650-665 (2018).

439 2. Lee, T. I. \& Young, R. A. Transcriptional Regulation and Its Misregulation in Disease.

$440 \quad$ Cell 152, 1237-1251 (2013).

441 3. Johnson, D. S., Mortazavi, A., Myers, R. M. \& Wold, B. Genome-wide mapping of in

442 vivo protein-DNA interactions. Science 316, 1497-1502 (2007).

443 4. McLean, C. Y. et al. GREAT improves functional interpretation of cis-regulatory

444 regions. Nat. Biotechnol. 28, 495-501 (2010).

445 5. Rosenfeld, M. G. Sensors and signals: a coactivator/corepressor/epigenetic code for 446 integrating signal-dependent programs of transcriptional response. Genes Dev. 20, 447 1405-1428 (2006). 
448 6. Thurman, R. E. et al. The accessible chromatin landscape of the human genome.

$449 \quad$ Nature 489, 75-82 (2012).

450 7. Buenrostro, J. D., Giresi, P. G., Zaba, L. C., Chang, H. Y. \& Greenleaf, W. J. Transposition

451 of native chromatin for fast and sensitive epigenomic profiling of open chromatin,

452 DNA-binding proteins and nucleosome position. Nat. Methods 10, 1213-1218

453 (2013).

454 8. Buenrostro, J. D. et al. Single-cell chromatin accessibility reveals principles of

455 regulatory variation. Nature 523, 486-490 (2015).

456 9. Wenger, A. M. et al. PRISM offers a comprehensive genomic approach to

457 transcription factor function prediction. Genome Res. 23, 889-904 (2013).

458 10. Ashburner, M. et al. Gene ontology: tool for the unification of biology. The Gene

459 Ontology Consortium. Nat. Genet. 25, 25-29 (2000).

460 11. The Gene Ontology Consortium. Expansion of the Gene Ontology

461 knowledgebase and resources. Nucleic Acids Res. 45, D331-D338 (2017).

462 12. Smith, C. L. \& Eppig, J. T. Expanding the mammalian phenotype ontology to

463 support automated exchange of high throughput mouse phenotyping data generated

464 by large-scale mouse knockout screens. J. Biomed. Semant. 6, 11 (2015).

465 13. Gerondakis, S. \& Siebenlist, U. Roles of the NF-кB Pathway in Lymphocyte

466 Development and Function. Cold Spring Harb. Perspect. Biol. 2, a000182 (2010).

467 14. Pikkarainen, S., Tokola, H., Kerkelä, R. \& Ruskoaho, H. GATA transcription

468 factors in the developing and adult heart. Cardiovasc. Res. 63, 196-207 (2004). 
469 15. Takahashi, K. \& Yamanaka, S. Induction of Pluripotent Stem Cells from Mouse

470 Embryonic and Adult Fibroblast Cultures by Defined Factors. Cell 126, 663-676

$471 \quad$ (2006).

472 16. Thiel, G. Transcription Factors in the Nervous System: Development, Brain

$473 \quad$ Function, and Diseases. (John Wiley \& Sons, 2006).

474 17. Hodge, R. D. \& Hevner, R. F. Expression and actions of transcription factors in

475 adult hippocampal neurogenesis. Dev. Neurobiol. 71, 680-689 (2011).

476 18. Maaten, L. V. D. \& Hinton, G. Visualizing Data using t-SNE. J. Mach. Learn. Res. 9, $477 \quad 2579-2605$ (2008).

478 19. DeKoter, R. P. et al. Regulation of Follicular B Cell Differentiation by the Related

479 E26 Transformation-Specific Transcription Factors PU.1, Spi-B, and Spi-C. J.

480 Immunol. 1001413 (2010). doi:10.4049/jimmunol.1001413

481 20. Sokalski, K. M. et al. Deletion of genes encoding PU.1 and Spi-B in B cells impairs

482 differentiation and induces pre-B cell acute lymphoblastic leukemia. Blood blood-

483 2011-02-335539 (2011). doi:10.1182/blood-2011-02-335539

484 21. Willis, S. N. et al. Environmental sensing by mature B cells is controlled by the

485 transcription factors PU.1 and SpiB. Nat. Commun. 8, 1426 (2017).

486 22. Woolf, E. et al. Runx3 and Runx1 are required for CD8 T cell development

487 during thymopoiesis. Proc. Natl. Acad. Sci. U. S. A. 100, 7731-7736 (2003).

488 23. Calderon, D. et al. Landscape of stimulation-responsive chromatin across

489 diverse human immune cells. bioRxiv 409722 (2018). doi:10.1101/409722

490 24. Loh, K. M. et al. Mapping the Pairwise Choices Leading from Pluripotency to

491 Human Bone, Heart, and Other Mesoderm Cell Types. Cell 166, 451-467 (2016). 
492 25. Papaioannou, V. E. The T-box gene family: emerging roles in development, stem $493 \quad$ cells and cancer. Development 141, 3819-3833 (2014).

494 26. Weidgang, C. E. et al. TBX3 Directs Cell-Fate Decision toward Mesendoderm. 495 Stem Cell Rep. 1, 248-265 (2013).

496 27. Denans, N., Iimura, T. \& Pourquié, O. Hox genes control vertebrate body

497 elongation by collinear Wnt repression. eLife (2015). doi:10.7554/eLife.04379

498 28. Machon, O., Masek, J., Machonova, O., Krauss, S. \& Kozmik, Z. Meis2 is essential 499 for cranial and cardiac neural crest development. BMC Dev. Biol. 15, 40 (2015).

$500 \quad 29 . \quad$ Inoue, T., Ota, M., Mikoshiba, K. \& Aruga, J. Zic2 and Zic3 synergistically control 501 neurulation and segmentation of paraxial mesoderm in mouse embryo. Dev. Biol. $502 \quad 306,669-684(2007)$.

503 30. Rojas, A. et al. Gata4 expression in lateral mesoderm is downstream of BMP4 504 and is activated directly by Forkhead and GATA transcription factors through a 505 distal enhancer element. Development 132, 3405-3417 (2005).

506 31. Park, B.-Y. \& Saint-Jeannet, J.-P. Expression analysis of Runx3 and other Runx 507 family members during Xenopus development. Gene Expr. Patterns GEP 10, 159-166 $508 \quad$ (2010).

509 32. Kalev-Zylinska, M. L. et al. Runx3 is required for hematopoietic development in $510 \quad$ zebrafish. Dev. Dyn. 228, 323-336 (2003).

511 33. Tsiairis, C. D. \& McMahon, A. P. A Hh-dependent Pathway in Lateral Plate 512 Mesoderm Enables The Generation Of Left-Right Asymmetry. Curr. Biol. CB 19, 513 1912-1917 (2009). 
514 34. Jahangiri, L. et al. The AP-1 transcription factor component Fosl2 potentiates

515 the rate of myocardial differentiation from the zebrafish second heart field. Dev.

516 Camb. Engl. 143, 113-122 (2016).

517 35. Dyer, L. A. \& Kirby, M. L. Sonic hedgehog maintains proliferation in secondary

518 heart field progenitors and is required for normal arterial pole formation. Dev. Biol.

$519 \quad 330,305-317(2009)$.

520 36. Tsokos, G. C. Systemic Lupus Erythematosus. N. Engl. J. Med. 365, 2110-2121

$521 \quad$ (2011).

522 37. Scharer, C. D. et al. ATAC-seq on biobanked specimens defines a unique

523 chromatin accessibility structure in naïve SLE B cells. Sci. Rep. 6, 27030 (2016).

524 38. Fujikura, D. et al. Death receptor 6 contributes to autoimmunity in lupus-prone 525 mice. Nat. Commun. 8, 13957 (2017).

526 39. Qin, S. et al. The Effect of SHH-Gli Signaling Pathway on the Synovial Fibroblast 527 Proliferation in Rheumatoid Arthritis. Inflammation 39, 503-512 (2016).

528 40. Bakhtadze, E. et al. Common variants in the TCF7L2 gene help to differentiate 529 autoimmune from non-autoimmune diabetes in young (15-34 years) but not in 530 middle-aged (40-59 years) diabetic patients. Diabetologia 51, 2224-2232 (2008).

531 41. Stridh, P. et al. Fine-Mapping Resolves Eae23 into Two QTLs and Implicates 532 ZEB1 as a Candidate Gene Regulating Experimental Neuroinflammation in Rat. PLOS 533 ONE 5, e12716 (2010).

534 42. van den Brink, S. C. et al. Single-cell sequencing reveals dissociation-induced 535 gene expression in tissue subpopulations. Nat. Methods 14, 935-936 (2017). 
536 43. Van Esch, H. \& Bilous, R. W. GATA3 and kidney development: why case reports

537 are still important. Nephrol. Dial. Transplant. 16, 2130-2132 (2001).

538 44. Kreidberg, J. A. WT1 and kidney progenitor cells. Organogenesis 6, 61-70

539 (2010).

$540 \quad 45 . \quad$ Park, C., Kim, T. M. \& Malik, A. B. Transcriptional regulation of endothelial cell

541 and vascular development. Circ. Res. 112, 1380-1400 (2013).

542 46. Eckert, R. L. et al. AP1 transcription factors in epidermal differentiation and skin 543 cancer. J. Skin Cancer 2013, 537028 (2013).

544 47. Regev, A. et al. The Human Cell Atlas. eLife 6, e27041 (2017).

545 48. Zerbino, D. R. et al. Ensembl 2018. Nucleic Acids Res. 46, D754-D761 (2018).

546 49. Köhler, S. et al. Expansion of the Human Phenotype Ontology (HPO) knowledge

547 base and resources. Nucleic Acids Res. (2018). doi:10.1093/nar/gky1105

$548 \quad 50 . \quad$ Kent, W. J. et al. The human genome browser at UCSC. Genome Res. 12, 996$5491006(2002)$.

550 51. Guturu, H., Doxey, A. C., Wenger, A. M. \& Bejerano, G. Structure-aided prediction 551 of mammalian transcription factor complexes in conserved non-coding elements.

552 Philos. Trans. R. Soc. Lond. B. Biol. Sci. 368, 20130029 (2013).

553 52. Tange, O. GNU Parallel - The Command-Line Power Tool. Login USENIX Mag. 36, $554 \quad 42-47(2011)$

555 53. Cormen, T. H., Leiserson, C. E., Rivest, R. L. \& Stein, C. Introduction to Algorithms, $556 \quad 3 r d$ Edition. (The MIT Press, 2009).

557 54. ENCODE Project Consortium. An integrated encyclopedia of DNA elements in 558 the human genome. Nature 489, 57-74 (2012). 
559 55. Roadmap Epigenomics Consortium et al. Integrative analysis of 111 reference

560 human epigenomes. Nature 518, 317-330 (2015).

561 56. Pedregosa, F. et al. Scikit-learn: machine learning in python.J Mach Learn Res

$562 \quad 12,2825-2830(2011)$.

563 57. Quinlan, A. R. \& Hall, I. M. BEDTools: a flexible suite of utilities for comparing

564 genomic features. Bioinformatics 26, 841-842 (2010).

565 58. Langmead, B. \& Salzberg, S. L. Fast gapped-read alignment with Bowtie 2. Nat.

$566 \quad$ Methods 9, 357-359 (2012).

567 59. Li, H. et al. The Sequence Alignment/Map format and SAMtools. Bioinforma. Oxf.

568 Engl. 25, 2078-2079 (2009).

569 60. Zhang, Y. et al. Model-based Analysis of ChIP-Seq (MACS). Genome Biol. 9, R137

$570 \quad$ (2008).

571 61. Li, X. et al. The impact of rare variation on gene expression across tissues.

$572 \quad$ Nature 550, 239-243 (2017).

573 


\section{Acknowledgements}

575 We thank Stanford's Kyle M. Loh, as well as Heidi Chen, Alex M. Tseng, and other

576 members of the Bejerano Lab for useful discussions, feedback and advice. Y.T. is supported

577 by a Funai Overseas Scholarship from the Funai Foundation for Information Technology

578 and the Stanford University School of Medicine. E.S.D. was supported in part by the

579 Simons Collaboration Grant on the Non-Perturbative Bootstrap. This work was supported

580 by National Institute of Mental Health (NIMH) of the National Institutes of Health (NIH)

581 under awards U01MH105949 to G.B. The content is solely the responsibility of the authors

582 and does not necessarily represent the official views of the National Institutes of Health.

\section{Author information}

\section{Author contributions}

585 E.S.D., Y.T. and G.B. conceived and designed the study. Y.T. updated GREAT. E.S.D.

586 conceived of and developed the WhichTF algorithm with support from Y.T. and G.B. E.S.D.

587 and Y.T. performed the computational analyses. Y.T. led the completion of the manuscript

588 with support from E.S.D. and oversight from G.B. Y.T. and E.S.D. contributed equally to

589 the project and author list is ordered by age. The manuscript was written and approved by all

590 authors.

\section{Competing interests}

592 The authors declare no competing interests. 
594 Figures and Tables

$595 \quad$ Figure 1

596

a WhichTF maps context-specific TFBSs to ontology functional terms (1) GREAT
regulatory domains
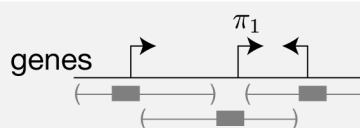

$\mathrm{TF}_{1}$

(2) PRISM

TFBSs predictions

(3) Query

(4) WhichTF

$$
\mathrm{TF}_{2}
$$$$
\text { : }
$$

$$
\mathrm{TF}_{\mathrm{N}}
$$
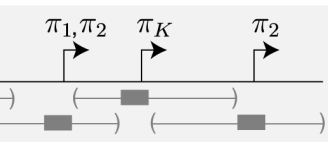
b TF x functional term
GREAT enrichment

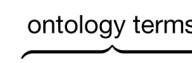

$\pi_{1} \pi_{2} \cdots \pi_{K}$

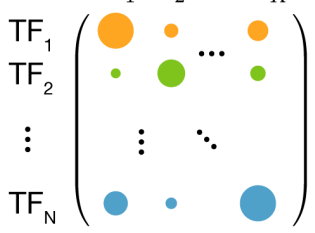

\section{$\Gamma$}

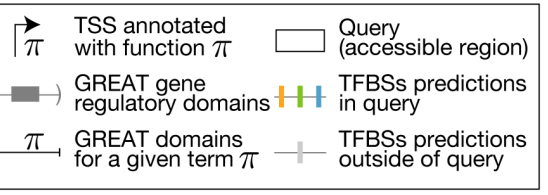

597 Fig. 1 WhichTF identifies dominant TFs for given experimental measurements of chromatin accessibility. (a) WhichTF uses gene regulatory domain models and ontologies from the genomic region enrichment analysis tool (GREAT) (step 1) and conservation-based PRISM predictions of TFBSs (step 2). Given a user-defined set of genomic regions (step 3),

601 WhichTF considers the top-K GREAT functional terms $\left(\pi_{1}, \ldots \pi_{K}\right)$ enriched in the query regions. For all pairwise combinations of top- $K$ term and TF, WhichTF counts the number of TFBSs within the specified query regions (step 4). (b) The binomial and hypergeometric

604 TFBS enrichment $p$-values for each ontology term are compiled in a TF-by-term summary

605 statistic matrix. (c) Aggregating the summary statistics over terms, WhichTF returns a

606 ranked list of TFs, ordered by predicted functional importance in the user-specific chromatin

607 environment, with the corresponding scores and statistics (Online Methods). TSS,

608 transcription start site. 
609 Figure 2

\begin{tabular}{|c|c|c|c|c|c|c|c|c|}
\hline & \multirow[b]{2}{*}{ TF } & \multicolumn{3}{|c|}{ B cells (ENCFF719GOE) } & \multirow[b]{2}{*}{ TF } & \multicolumn{3}{|c|}{ T cells (ENCFF861OSQ) } \\
\hline & & $-\log (\mathrm{CP})$ & Importance & PMID & & $-\log (\mathrm{CP})$ & Importance & PMID \\
\hline 1 & SPIB & 76.0 & Confirmed & 21057087 & NFKB1 & 96.8 & Confirmed & 20452952 \\
\hline 2 & NFKB1 & 89.6 & Confirmed & 20452952 & RUNX3 & 89.2 & Confirmed & 12796513 \\
\hline 3 & RELB & 62.1 & Confirmed & 20452952 & RELB & 63.5 & Confirmed & 20452952 \\
\hline 4 & RELA & 32.1 & Confirmed & 20452952 & RELA & 43.0 & Confirmed & 20452952 \\
\hline \multirow[t]{3}{*}{5} & SPIC & 11.5 & Confirmed & 21057087 & REL & 15.5 & Confirmed & 20452952 \\
\hline & & \multicolumn{3}{|c|}{ Heart (ENCFF176HSL) } & & \multicolumn{3}{|c|}{ Brain (ENCFF318HIS) } \\
\hline & TF & $-\log (\mathrm{CP})$ & Importance & PMID & TF & $-\log (C P)$ & Importance & PMID \\
\hline 1 & GATA5 & 50.5 & Confirmed & 16987437 & SOX2 & 69.4 & Confirmed & 28733588 \\
\hline 2 & GATA4 & 19.5 & Confirmed & 16987437 & OTX1 & 12.5 & Confirmed & 20354145 \\
\hline 3 & GATA6 & 18.3 & Confirmed & 28178271 & GLI1 & 16.8 & Confirmed & 14581620 \\
\hline 4 & TEAD4 & 10.8 & Confirmed & 16987437 & GLI2 & 7.9 & Confirmed & 14581620 \\
\hline 5 & FOS & 12.1 & Confirmed & 16934006 & ISL1 & 6.8 & Confirmed & 24763339 \\
\hline
\end{tabular}

612

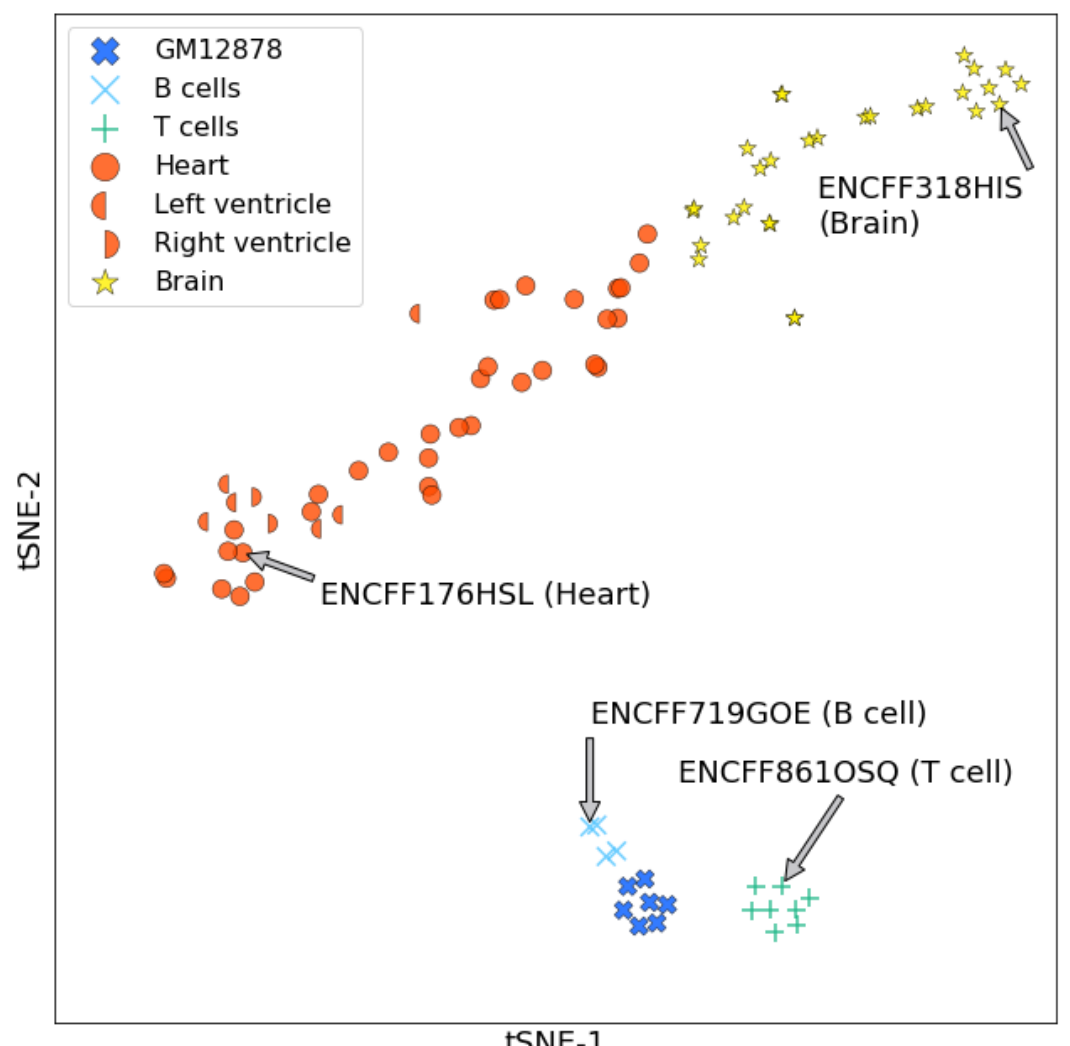

613 Fig. 2 WhichTF identifies dominant TFs in diverse cell types. (a) The top 5 identified

614 dominant TFs for B-, T-, heart, and brain cells are shown with the corresponding negative 
$615 \log$ conditional probability $(-\log \mathrm{CP})$, a statistical significance of the score of each $\mathrm{TF}$,

616 conditioned on the TFs with higher score (Online Methods). The importance and PubMed

617 ID (PMID) columns indicate whether existing literature supports the role of the identified

618 TFs, typically through perturbation experiments. (b) For DNase-seq data tracks of 90

619 samples across 7 cell types, the WhichTF score vectors are projected to t-SNE plot.

620 WhichTF quantitatively and robustly captures biological similarities and dissimilarities of

621 TF-mediated transcriptional programs. The samples highlighted in (a) are annotated with

622 arrows.

623 


\section{Figure 3}

$625 \mathbf{a}$

626
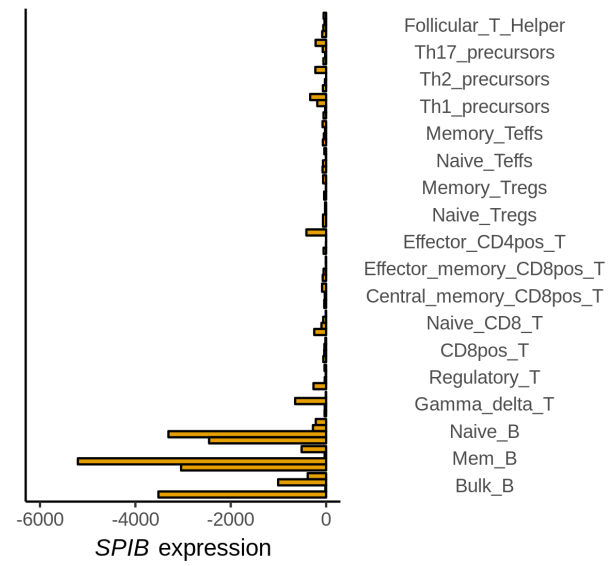

Follicular_T_Helper

Th17_precursors

Th2 precursors

Th1_precursors

Memory_Teffs

Naive_Teffs

Memory_Tregs

Naive Tregs

Effector_CD4pos_T

Effector_memory_CD8pos_T

Central_memory_CD8pos_T

Naive_CD8_T

CD8pos_T

Regulatory_T

Gamma_delta_T

Naive_B

Mem_B

Bulk_B

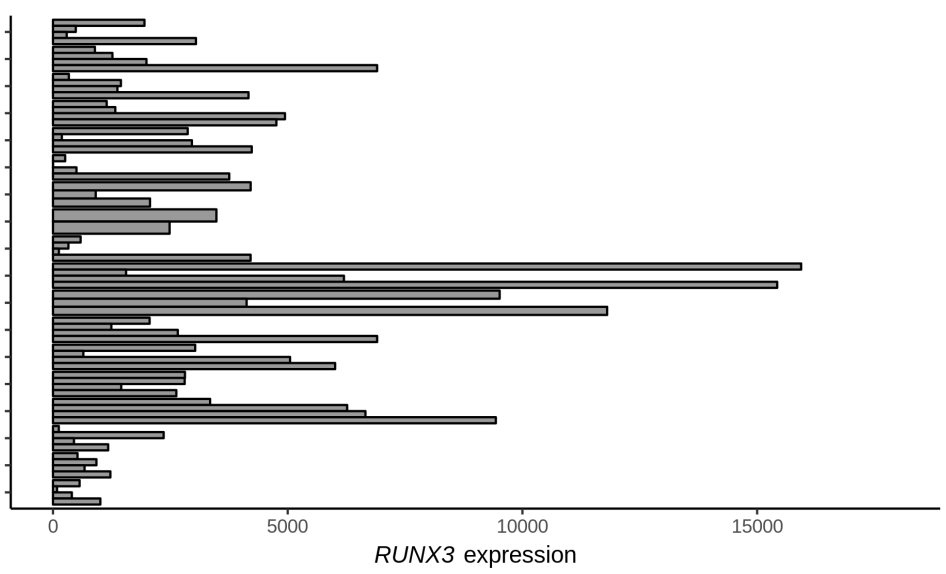

627 b

\begin{tabular}{llrll|lrll}
\hline \multicolumn{5}{c|}{ B cells - T cells } & \multicolumn{4}{c}{ T cells - B cells } \\
& TF & $-\log (\mathrm{CP})$ & Importance & PMID & TF & -log(CP) & Importance & PMID \\
\hline 1 & SPIB & 28.4 & Confirmed & 21057087 & RUNX3 & 171.1 & Confirmed & 12796513 \\
2 & SPI1 & 21.4 & Confirmed & 21057087 & NFKB1 & 47.7 & Confirmed & 20452952 \\
3 & SPIC & 17.1 & Confirmed & 21057087 & RUNX1 & 36.5 & Confirmed & 12796513 \\
4 & REL & 4.3 & Confirmed & 20452952 & REL & 8.0 & Confirmed & 20452952 \\
5 & RELB & 2.8 & Confirmed & 20452952 & CBFB & 9.1 & Confirmed & 17185462 \\
\hline
\end{tabular}

628

629 Fig. 3 WhichTF identifies differentially dominant TFs in B and T-cell DNase-seq data. (a)

630 Gene expression of the top differential TF genes, $S P I-B$ and $R U N X 3$, are shown (horizontal

631 axis) across diverse lymphoid cell types (vertical axis) for up to four healthy donors. (b) The

632 top 5 differential TFs for B-cells relative to T-cells (B-cell - T-cell) and vice versa (T-cell -

633 B-cell) are shown with the corresponding statistical significance, negative log conditional

634 probabilities $(-\log \mathrm{CP})$. The importance and PubMed ID (PMID) columns indicate whether

635 existing literature supports the identified TFs.

636 
637 Figure 4

\begin{tabular}{|c|c|c|c|c|c|c|c|c|c|c|}
\hline & & & & & \multicolumn{6}{|l|}{ ESCs } \\
\hline \multirow[t]{2}{*}{$\mathbf{a}$} & \multicolumn{4}{|c|}{ Anterior primitive streak (APS) } & & \multirow[t]{2}{*}{ b } & \multicolumn{4}{|c|}{ Mid primitive streak (MPS) } \\
\hline & TF & $-\log (C P)$ & Imp. & PMID & & & TF & $-\log (C P)$ & Imp. & PMID \\
\hline 1 & RUNX3 & 11.7 & $S$ & 29899136 & & 1 & TCF7L2 & 16.9 & $\mathrm{C}$ & 27419872 \\
\hline 2 & TCF7L2 & 10.0 & $\mathrm{C}$ & 27419872 & & 2 & TBX2 & 13.0 & $S$ & 24319661 \\
\hline 3 & TBX3 & 7.4 & $\mathrm{C}$ & 24319661 & & 3 & TBR1 & 12.4 & $\mathrm{~N}$ & \\
\hline 4 & CRX & 7.5 & $S$ & 17440610 & & 4 & TBX15 & 13.0 & $\mathrm{~N}$ & \\
\hline 5 & LEF1 & 7.6 & $\mathrm{C}$ & 27419872 & & 5 & GATA4 & 6.2 & $\mathrm{~S}$ & 27419872 \\
\hline \multirow[t]{2}{*}{ c } & \multicolumn{4}{|c|}{ Paraxial mesoderm } & & \multirow[t]{2}{*}{$\mathbf{e}$} & \multicolumn{4}{|c|}{ Lateral mesoderm } \\
\hline & TF & $-\log (C P)$ & Imp. & PMID & & & TF & $-\log (C P)$ & Imp. & PMID \\
\hline 1 & TCF7L2 & 7.7 & $\mathrm{C}$ & 27419872 & & 1 & RUNX3 & 8.6 & $\mathrm{C}$ & 20433948 \\
\hline 2 & LEF1 & 6.9 & C & 27419872 & & 2 & GLI1 & 10.9 & C & 19879143 \\
\hline 3 & $\mathrm{HOXC13}$ & 4.8 & $\mathrm{C}$ & 25719209 & & 3 & GATA4 & 7.1 & $\mathrm{C}$ & 27419872 \\
\hline 4 & TCF71 & 4.6 & $\mathrm{C}$ & 27419872 & & 4 & GATA6 & 5.1 & C & 27419872 \\
\hline 5 & IKZF3 & 3.6 & $\mathrm{~N}$ & & & 5 & GATA2 & 4.7 & $\mathrm{C}$ & 27419872 \\
\hline \multirow[t]{2}{*}{ d } & \multicolumn{4}{|c|}{ Early somite } & & \multirow[t]{2}{*}{$f$} & \multicolumn{4}{|c|}{ Cardiac mesoderm } \\
\hline & TF & $-\log (C P)$ & Imp. & PMID & & & TF & $-\log (C P)$ & Imp. & PMID \\
\hline 1 & MEIS2 & 6.9 & $\mathrm{C}$ & 9337138 & & 1 & GLI1 & 10.9 & $\mathrm{C}$ & 23873040 \\
\hline 2 & ZIC2 & 6.6 & $\mathrm{C}$ & 17490632 & & 2 & FOS & 6.7 & $\mathrm{C}$ & 11003651 \\
\hline 3 & INSM1 & 4.3 & $S$ & 25053427 & & 3 & GATA4 & 7.8 & $\mathrm{C}$ & 24790981 \\
\hline 4 & TEAD4 & 4.1 & C & 29636889 & & 4 & FOSL2 & 6.5 & C & 26732840 \\
\hline 5 & FOXI1 & 3.9 & $\mathrm{~N}$ & & & 5 & GATA1 & 5.2 & $\mathrm{C}$ & 21464046 \\
\hline
\end{tabular}

638

639 Fig. 4 WhichTF identifies differentially dominant TFs compared to immediate progenitor

640 cells along human mesoderm development pathway from ATAC-seq data. The top 5 TFs

641 with the corresponding statistical significance, negative log conditional probabilities (-log

642 CP) are shown. The importance (Imp.) and PubMed ID (PMID) columns indicate whether

643 (i) existing literature supports the identified TFs (C: confirmed); (ii) literature reports

644 closely related factors, such as co-factors and functionally related family members, or the

645 identified TFs in related context (S: suggestive); or (iii) novel (N). ESCs, embryonic stem

646 cells. 
647 Table 1: WhichTF identifies disease relevant TFs

\begin{tabular}{llrlr|lrlr}
\hline & \multicolumn{4}{c|}{ HC - SLE } & \multicolumn{4}{c}{ SLE - HC } \\
& TF & $-\log (\mathrm{CP})$ & Imp. & PMID & TF & $-\log (\mathrm{CP})$ & Imp. & PMID \\
\hline 1 & BCL6 & 28.7 & $\mathrm{C}$ & 28045014 & GLI1 & 19.7 & $\mathrm{~S}$ & 26552406 \\
2 & TFAP2B & 19.3 & $\mathrm{~N}$ & & ZFP143 & 11.0 & $\mathrm{~N}$ & \\
3 & ZEB1 & 16.6 & $\mathrm{~S}$ & 20856809 & TCF7L2 & 6.0 & $\mathrm{~S}$ & 18839133 \\
4 & ZSCAN21 & 15.2 & $\mathrm{~N}$ & & ONECUT2 & 5.2 & $\mathrm{~S}$ & 28317889 \\
5 & ZSCAN20 & 14.2 & $\mathrm{~N}$ & & DMRTC2 & 3.8 & $\mathrm{~N}$ & \\
\hline
\end{tabular}

648

649 Table 1 WhichTF identifies differentially dominant TFs from ATAC-seq measurement of

650 B-cells from systemic lupus erythematosus (SLE) patients and healthy controls ( HC). The

651 top 5 TFs based on the analysis of HC with respect to SLE (HC - SLE) and vice versa (SLE

652 - HC) are shown with the corresponding statistical significance, negative log conditional

653 probabilities (- $\log$ CP). The importance (Imp.) and PubMed ID (PMID) columns indicate

654 whether literature supports the identified TFs: confirmed (C), suggestive (S), or novel (N). 
bioRxiv preprint doi: https://doi.org/10.1101/730200; this version posted August 20,2019 . The copyright holder for this preprint (which was not certified by peer review) is the author/funder, who has granted bioRxiv a license to display the preprint in perpetuity. It is made available under aCC-BY-NC 4.0 International license.

656 Table 2: WhichTF identifies stress response factors in different samples

657

\begin{tabular}{|c|c|c|c|c|c|c|c|c|c|c|c|c|c|c|c|c|c|c|}
\hline & & \multicolumn{3}{|c|}{ Keratinocyte } & \multicolumn{3}{|c|}{ Adrenal Gland } & \multicolumn{3}{|c|}{$\begin{array}{l}\text { Lymphatic Vessel } \\
\text { Endothelium } \\
\text { ENCFF354CZP }\end{array}$} & \multicolumn{3}{|c|}{$\begin{array}{c}\text { Pulmonary Artery } \\
\text { Endothelium } \\
\text { ENCFF596PRJ }\end{array}$} & \multicolumn{3}{|c|}{$\begin{array}{l}\text { Dermis Vessel } \\
\text { Endothelium } \\
\text { ENCFF908DMH }\end{array}$} \\
\hline 1 & SPIB & * & & FOSB & $*$ & + & ZFP410 & & & NFKB1 & & + & FOSL1 & & + & NFKB1 & & + \\
\hline 2 & NFKB1 & $*$ & + & FOS & $*$ & + & FOS & & + & FOS & & + & FOS & & + & FOS & & + \\
\hline 3 & RELB & $*$ & + & FOSL1 & $*$ & + & FOSL1 & & + & FOSL1 & & + & FOSL2 & & + & FOSL1 & & + \\
\hline 4 & RELA & $*$ & + & JUND & $*$ & + & NFKB1 & & + & RELB & & + & NFKB1 & & + & RELA & & + \\
\hline 5 & SPIC & $*$ & & BATF & & + & JUNB & & + & BATF & & + & JUND & & + & FOSL2 & & + \\
\hline 6 & SPI1 & $*$ & & FOSL2 & * & + & FOSL2 & & + & JUND & & + & RELB & & + & BATF & & + \\
\hline 7 & ZFP410 & & & BACH2 & & + & BACH1 & & + & FOSL2 & & + & BATF & & + & FOSB & & + \\
\hline 8 & RUNX3 & & & JUNB & * & + & JUND & & + & REL & & + & RELA & & + & RELB & & + \\
\hline 9 & REL & $*$ & + & BACH1 & & + & RELB & & + & RELA & & + & SOX10 & * & & JUND & & + \\
\hline 10 & STAT2 & $*$ & & JUN & * & + & $\mathrm{BACH} 2$ & & + & SPIC & $*$ & & FOSB & & + & SOX7 & * & \\
\hline 11 & WT1 & & & NFE2L2 & & & GATA3 & $*$ & & FOSB & & + & BACH2 & & + & ZFP410 & & \\
\hline 12 & SNAI3 & & & NFKB1 & & + & JUN & & + & ZFP410 & & & BACH1 & & + & BACH1 & & + \\
\hline 13 & ZEB2 & $*$ & & MZF1 & & & WT1 & * & & SPIB & $*$ & & GATA4 & $*$ & & GATA4 & * & \\
\hline 14 & ATF6 & & & RELB & & + & BATF & & + & SOX30 & $*$ & & JUNB & * & & SOX12 & $*$ & \\
\hline 15 & E2F5 & $*$ & & ZFP217 & & & NFE2L2 & & & SOX7 & * & & GATA5 & $*$ & & FOXD1 & * & \\
\hline 16 & IKZF3 & $*$ & & ETS2 & * & & GATA6 & * & & SOX18 & $*$ & & SOX 30 & * & & FOXJ3 & $*$ & \\
\hline 17 & ELF5 & & & PITX1 & & & FOSB & & + & JUNB & & + & SPIB & $*$ & & SOX30 & $*$ & \\
\hline 18 & SP100 & & & ATF6 & & & GATA4 & * & & SOX12 & $*$ & & SOX18 & $*$ & & SOX18 & $*$ & \\
\hline 19 & IRF9 & $*$ & & TFCP2L1 & & & MITF & * & & BACH1 & & + & JUN & $*$ & & FOXO6 & * & \\
\hline 20 & SNAI1 & & & MYC & * & & FOXP2 & * & & FOXO3 & $*$ & & FOXO3 & * & & FOXO4 & $*$ & \\
\hline
\end{tabular}

658 Table 2 WhichTF identifies TFs known for stress response. The top 20 TFs identified by

659 WhichTF are shown in ranked order for B-cells, keratinocytes, adrenal gland, lymphatic

660 vessel endothelium, pulmonary artery endothelium, and dermis vessel endothelium cells.

661 The TFs known to be involved in stress response signals are marked with plus (+), while

662 TFs in families known to be functionally important in each context are marked with asterisk

$663(*)$. 
665 Supplementary materials

666 List of supplementary materials

667 Supplementary Figures

668 - Supplementary Figure S1: Gene expression profile of TBX15

669 Supplementary tables

670 - Supplementary Table S1: Baseline TF enrichment method

671 - Supplementary Table S2: Mouse ENCODE dataset analysis

672 - Supplementary Table S3: The update summary of GREAT ontologies

673 - Supplementary Table S4: Human ENCODE datasets

674 - Supplementary Table S5: Mouse ENCODE datasets

675 - Supplementary Table S6: Mesoderm development samples

676 - Supplementary Table S7: Sequence read archive accession IDs for systemic

677 lupus erythematosus dataset

678 


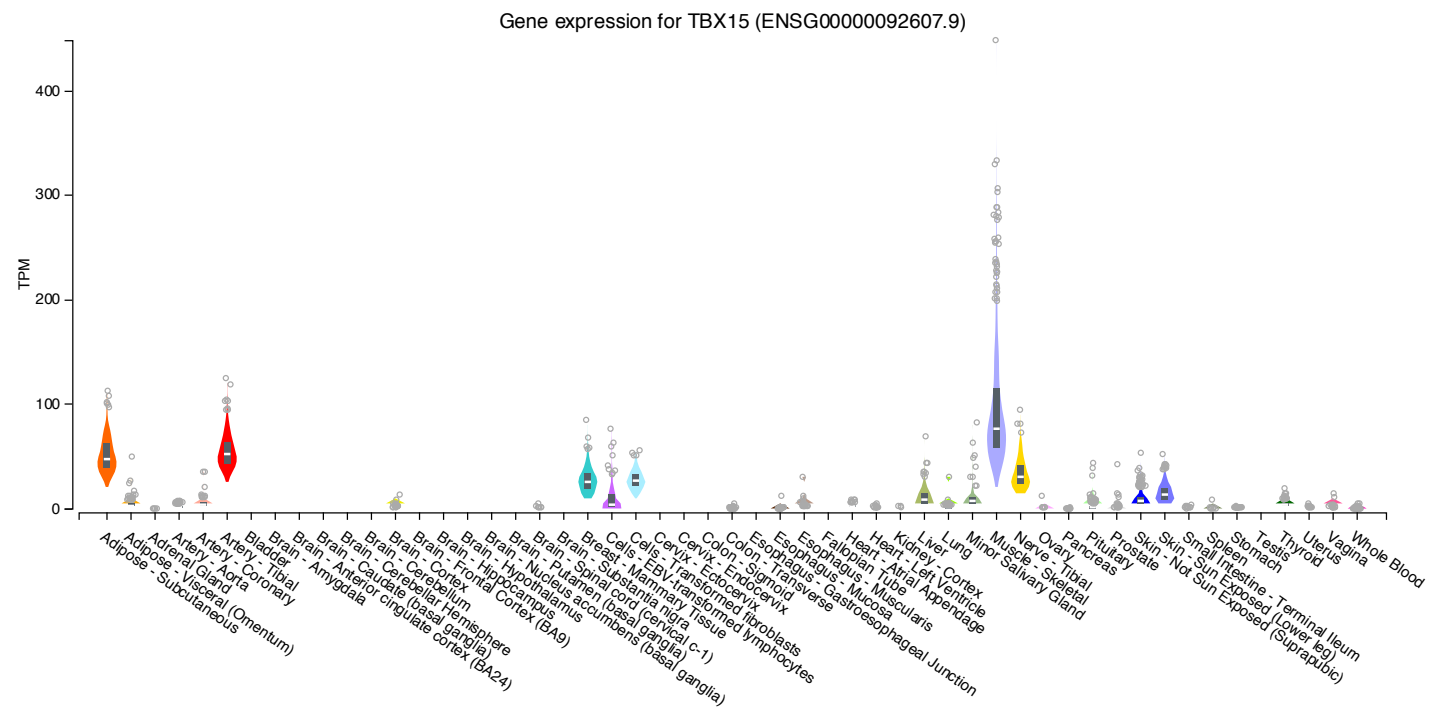

681 Supplementary Figure S1. Tissue-specific gene expression profile of $T B X 15$ in muscle.

682 The Human cell types are shown on $\mathrm{x}$-axis and the expression (TPM) is shown on y-axis.

683 The median and 25th and 75th percentiles are shown as box plots and data points are shown

684

685 as outliers if they are above or below 1.5 times the interquartile range.

\section{Supplementary Tables}

688 from ENCODE and Roadmap DNase-seq datasets are shown. The top 5 identified TFs are

689 shown for (a) B-cells, (b) T-cells, (c) heart cells, and (d) brain cells. ENCODE accession

690 IDs for each sample and the dominant TFs and their corresponding - $\log 10(\mathrm{p}$-value) are

691 shown. There is less cell-type specificity in the identified results.

692

693 Supplementary Table S2. Mouse ENCODE dataset analysis. WhichTF identifies dominant

694 TFs for four mouse cell types from ENCODE and Roadmap DNase-seq dataset. The top 5

695 identified dominant TFs are shown for (a) B-cells, (b) T-cells, (c) heart cells, and (d)

696 hindbrain cells. The ENCODE accession IDs for each sample are shown on the top and the 
697 dominant TFs and their corresponding statistical significance, conditional probabilities, are

698 shown.

699

700 Supplementary table S3. The update summary of GREAT ontologies. Ensembl genes is a

701 flat ontology defined from the set of genes with at least one meaningful annotation in gene

702 ontology (Online Methods). GO: gene ontology. HPO: human phenotype ontology. MGI:

703 mouse genome informatics.

704

705 Supplementary Table S4. Human ENCODE datasets. The list of ENCODE accession IDs

706 used in our study and the corresponding cell type or tissues.

707

708 Supplementary Table S5. Mouse ENCODE datasets. The list of ENCODE accession IDs

709 used in our study and the corresponding cell type or tissues.

710

711 Supplementary Table S6. Mesoderm development samples. The list of sample IDs, sample

712 description, and the reference to the corresponding results.

713

714 Supplementary Table S7. Sequence read archive (SRA) accession IDs for systemic lupus

715 erythematosus dataset. SLE indicates disease and HC indicates healthy control. 